\title{
Morphology Portion or Totality
}

National Cancer Institute

\section{Source}

National Cancer Institute. Morphology Portion or Totality. NCI Thesaurus. Code

C117623.

A qualifier for the arrangement or apportioning of an entity within a body part, region or organ for a morphology assessment. 